\title{
Laboreal
}

Volume $16 \mathrm{~N}^{\circ} 2$ | 2020

Programa de Pesquisa do Curso da Ação

\section{A construção conjunta de um observatório e de um objeto teórico : o exemplo do relato da experiência e do espaço de ação}

La construcción conjunta de un observatorio y de un objeto teórico : el ejemplo del relato de experiencia y del espacio de acción La construction conjointe d'un observatoire et d'un objet théorique : l'exemple du récit d'expérience et de l'espace d'actions The joint construction of an observatory and a theoretical object : an example with the story of experience and the field of action

Nicolas Terré, Carole Sève y Benoît Huet

\section{OpenEdition}

\section{Journals}

Edición electrónica

URL: http://journals.openedition.org/laboreal/16942

DOI: $10.4000 /$ laboreal. 16942

ISSN: 1646-5237

Editor

Universidade do Porto

Referencia electrónica

Nicolas Terré, Carole Sève y Benoît Huet, « A construção conjunta de um observatório e de um objeto teórico : o exemplo do relato da experiência e do espaço de ação », Laboreal [En línea], Volume 16 NN2 $^{0}$ 2020, Publicado el 01 diciembre 2020, consultado el 13 diciembre 2020. URL : http:// journals.openedition.org/laboreal/16942; DOI : https://doi.org/10.4000/laboreal.16942

Este documento fue generado automáticamente el 13 diciembre 2020.

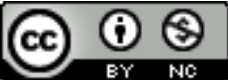

Laboreal está licenciado com uma Licença Creative Commons - Atribuição-NãoComercial 4.0 Internacional. 


\section{A construção conjunta de um observatório e de um objeto teórico : o exemplo do relato da experiência e do espaço de ação}

La construcción conjunta de un observatorio y de un objeto teórico : el ejemplo

del relato de experiencia y del espacio de acción

La construction conjointe d'un observatoire et d'un objet théorique : l'exemple

du récit d'expérience et de l'espace d'actions

The joint construction of an observatory and a theoretical object : an example

with the story of experience and the field of action

Nicolas Terré, Carole Sève y Benoît Huet

NOTA DEL EDITOR

Manuscrito recibido en : 02/03/2020

Aceptado tras peritaje en : 26/10/2020

Artigo publicado em língua francesa na revista Activités, vol. 17, n² de 2020 [Le

programme de recherche cours d'action]

Traducción : Adriana Elena Ramponi [aeramponi@gmail.com]

\section{Introducción}

Este artículo presenta la manera en la que hemos construido, durante una investigación sobre la enseñanza y el aprendizaje del kayak de mar en el ámbito escolar, un observatorio (centrado en relatos de experiencia), un objeto teórico (los espacios de acción como reducción del "curso de acción") y un objeto de estudio particular (la 
apropiación), todo ello, en coherencia con las hipótesis ontológicas, teóricas y metodológicas del Programa de Investigación del "curso de acción" (de aquí en adelante Programa CdA) . Para dar visibilidad a este proceso de construcción conjunta, y porque este caso especial nos brinda la oportunidad, elegimos explicar la historicidad de esta investigación. En efecto, aun cuando es habitual delimitar un objeto de estudio antes de optar por una situación de estudio en la cronología de un artículo científico, la realidad de la investigación puede ser completamente otra. Consiste, a veces, en tomar una situación para su estudio que, durante el proceso, puede convertirse en una situación de estudio vinculada a un objeto que estaba mal definido inicialmente. Es posible también que durante la investigación intervengan innovaciones metodológicas (en nuestro caso, los relatos de experiencia ) y teóricas (en nuestro caso, los espacios de acción). Para dar cuenta de esta dimensión rara vez develada de la investigación, que ilustra un Programa CdA abierto a la ingeniería de los observatorios y a los avancesrefutaciones teóricos (Theureau, 2006), este artículo se estructura en adelante de manera cronológica. Estimamos que esto puede explicar cómo desafíos teóricos y metodológicos emergen e invitan a idas y venidas entre el diseño del observatorio, la definición del objeto teórico, el procesamiento de los datos y la delimitación del objeto de estudio.

\section{El proyecto “kayak ártico” : una situación para estudiar}

2 Desde un principio, el proyecto escolar "Kayak ártico" (Terré \& Jan, 2019) resultó ser un imperativo para un estudio empírico. Desde hace trece años, dos profesores de EPS (Education Physique et Sportive, Educación Física y Deportiva), interesados en comprender de manera pormenorizada cómo se dan los aprendizajes de los alumnos, llevan adelante este proyecto escolar. Uno de estos profesores es además investigador y se involucró en este estudio. El proyecto es interdisciplinario y asocia a la EPS con otras disciplinas escolares. Se lo propone a alumnos voluntarios de un curso de "tercero" (alumnos de 14 a 15 años) y apunta a descubrir la cultura inuit. En las clases de EPS, se sensibiliza a los alumnos en esta cultura por medio del aprendizaje de técnicas originales del kayak de mar. El año comienza con talleres sobre el aprendizaje de diversas técnicas de esquimotaje (ej. : técnicas para volver a la superficie después de un vuelco, sin salir del kayak). Luego, sigue con el aprendizaje de la propulsión. Concluyen el año escolar con una estadía de dos días para navegar en las corrientes del Golfo de Morbihan [1]. Durante el año, los profesores de otras disciplinas aprovechan la oportunidad que les brinda este proyecto para abordar de manera diferente contenidos disciplinarios : autobiografía en el curso de Lengua a partir de las experiencias de los alumnos en el agua, trigonometría en Matemáticas a partir de las corrientes, acuarela en Artes Plásticas a partir de un texto de Jorn Riel, las sonoridades inuit en Educación Musical, energía cinética y normas de prioridad en el mar en Ciencias Físicas, etc.. Nos pareció interesante analizar los efectos particularmente visibles que este proyecto produce en los alumnos: marcados progresos en kayak de mar (el éxito de los esquimotajes en más de doscientos alumnos desde hace trece años; la soltura con la que hacen frente a las corrientes y contracorrientes del Golfo de Morbihan cuando las descubren por primera vez a fin de año), el enorme entusiasmo y la cohesión creciente en el seno de la clase durante todo el año. 
Inicialmente, lo que impulsó este estudio fue un interrogante de investigación relativamente amplio : ¿qué se juega en la actividad de los alumnos en relación con lo que les proponen sus profesores, y quién puede explicar sus progresos, su entusiasmo, su sentimiento de estar viviendo una aventura compartida? De entrada, dos orientaciones delimitaron este interrogante. La primera tiene que ver con la reducción de la actividad a la experiencia (Theureau, 2006), es decir a la parte de la actividad que resulta significativa para el actor ; dicho de otro modo, el flujo de fenómenos que están "presentes" para él en su actividad y que forman su "propio mundo" (por ejemplo, la experiencia de estar remando, de sentir un apoyo en el agua, de querer divertirse en las olas, de experimentar confianza frente a ciertos tipos de embarcación, de preguntarse acerca de la importancia de la inclinación del remo, de experimentar una cierta excitación frente al desafío de lograr realizar su primer esquimotaje, etc.). La segunda orientación es no reducir el aprendizaje a las simples transformaciones del sujeto. Adherimos a la definición del aprendizaje entendido como "un proceso continuo de las transformaciones conjuntas de un individuo y de un entorno, a partir de la historia de las diversas acciones y comunicaciones que lleva a cabo ese individuo en ese entorno" (Varela, Thompson, \& Rosch, 1993, p. 23, traducción libre). Esta definición del aprendizaje es la que nos condujo a investigar transformaciones en el acoplamiento entre el actor y su entorno. Entendemos que esta descentración del sujeto puede enriquecer una concepción enactiva del aprendizaje a través de una comprensión más aguda de los procesos de apropiación, y abrir "nuevos posibles" en materia de intervención en el campo de la formación y la enseñanza, y hasta en otros campos profesionales.

\section{Los relatos de experiencia : un observatorio de los cursos de la experiencia de un colectivo de alumnos durante un año}

El primer desafío era metodológico. Se trataba de concebir un observatorio para investigar sobre un colectivo de alumnos (veintiséis alumnos) y durante un lapso de tiempo relativamente largo (nueve clases distribuidas a lo largo de un año escolar para cada uno de los dos grupos).

\subsection{Un conjunto de posibles metodológicos en el Programa CdA}

El diseño del observatorio se inspiró primero en métodos utilizados frecuentemente en el Programa CdA, pero no directamente extrapolables al contexto de este estudio. En una corriente análoga a la de la experiencia del usuario (Cahour, 2013 ; Norman, 1988), consideramos a los alumnos como aliados tanto para la investigación como para la intervención. Mediando condiciones favorables, son capaces de revelar, a través de su vivencia, problemáticas de enseñanza-aprendizaje (Bolstad, 2011 ; Mitra, 2009).

Para ayudar a los actores en expresar su vivencia, el Programa CdA propone varios métodos que apuntan a tener en cuenta trazas de la actividad que ayuden al actor en ponerse en situación y contar, comentar, describir y mimar su actividad tal como resultaba significativa para él en el momento en que se desarrolló. Estos métodos tienen por objetivo recabar datos para documentar el acoplamiento actor-situación. Sin 
embargo, por lo menos tres razones no nos permitieron concebir el observatorio a partir de métodos habituales. La primera razón tiene que ver con la especificidad del lugar de práctica, que hace difícil la grabación de videos y audios en el agua, o el apoyo sobre trazas materiales. La segunda tiene relación con la cantidad de participantes en el estudio y la dificultad de ofrecer la posibilidad a veintiséis alumnos de participar en las entrevistas. Por último, la tercera razón remite a la duración del estudio y a la dificultad de perpetuar la organización de las entrevistas a lo largo de 16 clases (ocho clases por grupo), en todo un año escolar. A estas razones se les agrega también el proyecto de diseñar un observatorio que no perturbe el desarrollo del curso y que permita un procesamiento fácil del conjunto de los datos recabados.

7 Nos orientamos pues hacia variantes de entrevistas de "puesta en situación" que se apoyan solamente en las trazas que la actividad dejó en los cuerpos mismos de los actores para ayudarlos en verbalizar su conciencia prerreflexiva (Theureau, 2006). Considerado por Theureau como un "por lo menos esto" (es decir, un método a falta de algo mejor), este método de puesta en situación puede sin embargo conducir a "tesoros de imaginación en materia de procedimientos de reflexión para llegar a algo que no sea en parte inducido por los investigadores" (Theureau, 2010, p. 293, traducción libre). Los relatos de experiencia en los que se funda nuestro observatorio son, desde nuestro punto de vista, una variante de ese tipo de entrevista.

\subsection{Un conjunto de oportunidades de innovación metodológica en el contexto local e institucional.}

8 El observatorio fue diseñado en la interfaz de posibles metodológicos y de las especificidades del contexto local e institucional. Esta articulación participaba de una voluntad de construir, por parte del observatorio, relaciones entre investigación, enseñanza y aprendizaje o entre los investigadores, los profesores y los alumnos (ver conclusión). Así las cosas, las contingencias locales fueron tomadas en esta investigación como oportunidades de innovación metodológica.

9 Tomamos partido de fusionar el observatorio con hábitos vinculados al proyecto "Kayak Ártico". Era costumbre, en el desarrollo del proyecto, que después de cada sesión, un alumno redactara un texto relatando su experiencia y que ese relato de experiencia fuera publicado en el blog de la clase (www.asqajaq.fr). Este texto acompañaba una filmación de aproximadamente dos minutos realizada por los profesores. Mientras se realizaba esta investigación, nos apoyamos en el interés manifestado por los alumnos para contar lo que vivían para extender a los veintiséis alumnos la posibilidad de redactar, voluntariamente, un relato de experiencia después de cada clase/sesión. La consigna era redactar, por medio de un formulario en línea , un texto de 100 a 150 palabras aproximadamente, lo antes posible, después de la clase.

10 La elección de anclar el observatorio en los relatos de experiencia tenía estrecha relación con orientaciones institucionales del colegio. En efecto, el trabajo del relato de experiencia está vinculado a varias expectativas de formación. (Recuadro 1). 
Extractos de la Base Común de Competencias, Conocimientos y Cultura (Ministerio de Educación Nacional de Francia, 2015):

"la expresión y la comunicación por escrito y en soportes varios (papel, digital) de una historia, de una descripción, de un sentimiento, de un punto de vista, de un juicio argumentado";

"explicitar las emociones experimentadas (la precisión del vocabulario utilizado por el alumno para expresar lo que percibe y siente, la capacidad para explicitar lo que le gusta y lo que le disgusta, para justificar lo que siente, para tener en cuenta reacciones de los interlocutores)";

- "practicar diversas formas de escritura inventadas y argumentativas (elaborar un texto, un objeto, una imagen que dé cuenta de una experiencia, de una percepción o de una emoción, estar en condiciones de explicitar las elecciones efectuadas para expresarlas, relacionar la intención y la forma)".

Recuadro 1: Expectativas de formación del colegio, referidos a la escritura y a la experiencia. Box 1: Learning outcomes for written and experience.

Recuadro 3 : Evolución dei espacio de acción de Anna clase tras clase

\subsection{Precisiones sobre la metodología escogida para la redacción de los relatos de experiencia}

11 El relato de experiencia puede proporcionar datos pertinentes sobre el acoplamiento del actor y de su situación tomando ciertas precauciones metodológicas. De la misma forma que en una entrevista de autoconfrontación se esperaba que, en la redacción de los relatos de experiencia, los alumnos pudieran contar y comentar su actividad tal como la habían vivido, sin pretender analizarla, racionalizarla o justificarla a posteriori (Theureau, 2016). Por lo tanto, había que tener la precaución de no caer en otras formas de relatos (analíticos, imaginarios) mucho más valorizados en la escuela que lo anecdótico, lo sensible y lo fáctico. Se trataba de tomar en serio el hecho de que toda escritura se inscribe en un espacio socio-institucional particular (Coli, 2014). El de la escuela incluye sus propios códigos. Valoriza, por ejemplo, posturas "segundas" que perciben a la escritura como un pretexto para la imaginación y el análisis, a expensas de posturas "primeras" que perciben a la escritura como una descripción directa del pensamiento a la manera de un discurso oral interior (Joubert, 2018).

Para evitar estas confusiones y capacitar a los alumnos en la redacción de los relatos de experiencia se implementaron tres ayudas metodológicas. La primera de ellas, estaba en la consigna inicial. Se le indicaba a los alumnos: "para ayudarte a contar tu sesión, puedes basarte en lo que hiciste o en lo que quisiste hacer, lo que sentiste, lo que te dijiste a ti mismo, lo que te marcó, lo que esperabas. Utiliza prioritariamente el presente y cuenta lo que viviste realmente". Con esta consigna, se esperaba que los alumnos intentaran estar más presentes en la situación pasada vivida que en la situación de escritura actual. La segunda ayuda aportada por los profesores consistió en proporcionar a los alumnos puntos de referencia para estructurar sus relatos, cuidando no modificar sus contenidos. Inspirándose en la metodología del relato de experiencia en clínica de la educación (Lani-Bayle, 2006), se les indicó entonces, a partir de extractos de sus producciones, que los relatos resultaban mejores si eran fácticos (es decir, si relataban "hechos") y sensoriales (es decir, si relataban "lo que los hechos me producen"). Por último, la tercera ayuda apuntaba a acompañarlos en su primera redacción. Al 
comienzo del año, todos los alumnos redactaron su relato en el colegio, con la presencia de los docentes que encuadraban las clases de kayak de mar. Estos les ayudaron a comprender e implementar lo que era esperado de los relatos de experiencia. En esa oportunidad, los profesores invitaron a los alumnos, por ejemplo, a enriquecer su relato saliendo de la simple percepción visual (que puede conducir a una posición aperceptiva de observador, ver Depraz, Varela, \& Vermersch, 2011) para reconectar con otras impresiones sensoriales. Después de las clases subsiguientes, los alumnos redactaron sus relatos en sus casas, a partir de un formulario que se les puso en su espacio electrónico de trabajo. Para garantizar la autenticidad de los dichos, estas contribuciones se realizaron voluntariamente, sin meta de evaluación.

Cada alumno podía redactar hasta un máximo de ocho relatos. En los hechos, se crearon durante ese año escolar ciento veintisiete relatos (sesenta y nueve relatos del primer grupo, cincuenta y ocho relatos del segundo grupo). Como máximo, algunos alumnos redactaron siete relatos. Como mínimo, redactaron tres. En promedio, redactaron cinco.

\section{El análisis de los relatos : puesta en evidencia de un resumen de experiencias particularmente pertinente para documentar el compromiso y los representamens de los alumnos.}

El segundo desafío era a la vez teórico y metodológico. Se trataba de basarse en el contenido de los relatos para dar cuenta de la experiencia vivida por los alumnos (y de su evolución) durante las clases de kayak. Esto se pudo realizar gracias a la construcción conjunta de un método de análisis y de un objeto teórico. Al inicio, como lo muestra esta sección, el objeto teórico era relativamente genérico. Se trataba de documentar componentes del curso de la experiencia. Seguidamente (sección siguiente), la reducción del objeto teórico a los espacios de acción de los alumnos nos condujo a volver al método de análisis de los relatos para precisarlo.

\subsection{Relatos heurísticos para describir un resumen de la experiencia}

15 Según las clases, todos los relatos no fueron construidos de la misma forma por los alumnos. Algunos poseen un carácter muy sintético y muestran un sentimiento global. La mayoría, como en el extracto presentado en el Cuadro 1, hacen referencia a acontecimientos precisos. Estos dan cuenta de lo que fue sobresaliente para el alumno en momentos particulares de la clase/sesión.

16 Todos los relatos fueron procesados de manera que pudieran documentar, respecto de la sesión en cuestión, los elementos significativos de la experiencia reportados por los alumnos. El hecho de que uno de los investigadores fuera también profesor de EPS en este proyecto, y por lo tanto comprometido en el mismo ámbito que los alumnos, resultó ser una ayuda para la interpretación de los relatos y la documentación de su experiencia. En relación al Programa CdA nos apoyamos, en todo caso, sobre los seis componentes que se incorporan, a cada instante, en la experiencia de un actor (es decir, los seis componentes del signo hexádico $\left.{ }^{2}\right]$ : el compromiso (E), las anticipaciones (A), lo referencial (S), el representamen (R), la unidad de experiencia (U), el interpretante (I). 
A diferencia de los datos recabados a partir de las grabaciones continuas de la actividad y de las entrevistas de "puesta en situación" (por ejemplo, Terré, Saury, Sève, 2013, respecto de otro estudio llevado a cabo con alumnos en kayak de mar), los datos recabados a partir de los relatos no permitieron reconstruir paso a paso el curso de la experiencia de los actores. Los relatos se referían a trazas de la actividad que los alumnos habían memorizado mejor y/o que podían reconstituir más fácilmente. Estos relatos nos permitieron, en consecuencia, documentar elementos significativos de la experiencia, que en la de los alumnos podían ser persistentes (ej., un compromiso continuo a lo largo de toda la clase), sobresalientes (ej., representamens más impactantes que otros) o recurrentes (ej., acciones reproducidas en varios momentos de la sesión). A título de ejemplo, el cuadro que sigue documenta elementos significativos en la experiencia de Anna a partir de su primer relato de experiencia.

Cuadro 1 : Relato y componentes de la experiencia de Anna durante la primera sesión

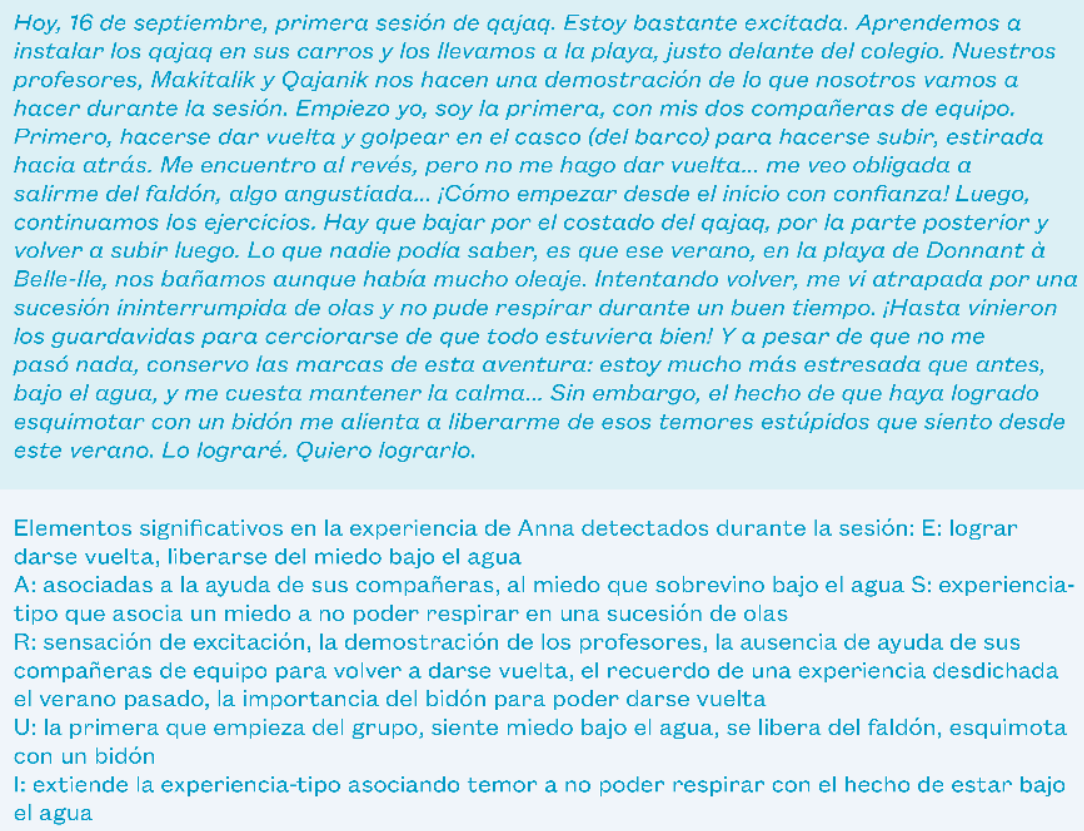

Cuadro 1 : Relato y componentes de la experiencia de Anna durante la primera sesión

\subsection{Relatos heurísticos para diferenciar el grado de compromiso de los alumnos}

17 Los relatos de experiencia resultaron fructíferos para explicar el compromiso del actor a un nivel relativamente macroscópico (a escala de la sesión). El compromiso corresponde a las preocupaciones y ocupaciones (o a las bifurcaciones en las preocupaciones y ocupaciones) más importantes para el alumno. Por ejemplo, en el relato de su primera clase, Anna contó que se había puesto como meta liberarse de su miedo (preocupación) tratando de darse vuelta, sola (ocupación). Los datos recabados a partir de los relatos de experiencia difieren en este punto con los recabados a partir de 
las entrevistas de "puesta en situación" que permiten documentar compromisos locales (referidos a la acción en curso) e identificar permanencias en el compromiso únicamente a partir de un procedimiento sintético-progresivo (Theureau \& Jeffroy, 1994). En los relatos de experiencia, contrariamente a las entrevistas de "puesta en situación", el alumno puede contar libremente su experiencia teniendo una visión global de la sesión, y no como si no conociera la historia en su totalidad.

La posibilidad de tener una primera visión sintética sobre el compromiso del actor respecto de la sesión nos parece particularmente heurístico en el campo de la formación y de la enseñanza. Esto da pautas sobre las historias (una ocupación que dura en el tiempo) en las que los alumnos están comprometidos (ej., lograr su primer esquimotaje antes de terminar la sesión) y sobre el modo de compromiso o preocupaciones típicas (ej., desafiarse unos con otros dentro del grupo de alumnos). Ahora bien, la naturaleza de los conocimientos construidos y la propensión a volver a movilizarlos están fuertemente vinculados a las historias, como pudimos verlo en un estudio en el campo del escalamiento (Terré, Sève, \& Saury, 2016). Por ejemplo, los alumnos tienden a construir conocimientos "endémicos" (es decir, cuyas oportunidades de actualización son reducidas) cuando se comprometen en historias que se desarrollan en torno a una ocupación y a una temporalidad limitadas a los dispositivos de aprendizaje particulares. Los alumnos también tienen tendencia en construir conocimientos más "robustos" (es decir, cuyas oportunidades de actualización son importantes) cuando se comprometen en historias que se desarrollan en torno a un modo de compromiso y de temporalidad que van más allá de los dispositivos de aprendizaje o de las clases.

Esta visión sintética sobre el compromiso de los alumnos resulta también interesante cuando el estudio de los relatos de experiencia se extiende al conjunto de los alumnos del curso ya que permite, por ejemplo, identificar las diferentes ocupaciones compartidas por ellos a lo largo de una sesión (ejemplo, Figura 1) o de varias sesiones. 
Figura 1 : Compromiso de los alumnos dei grupo 2 durante la primera sesión

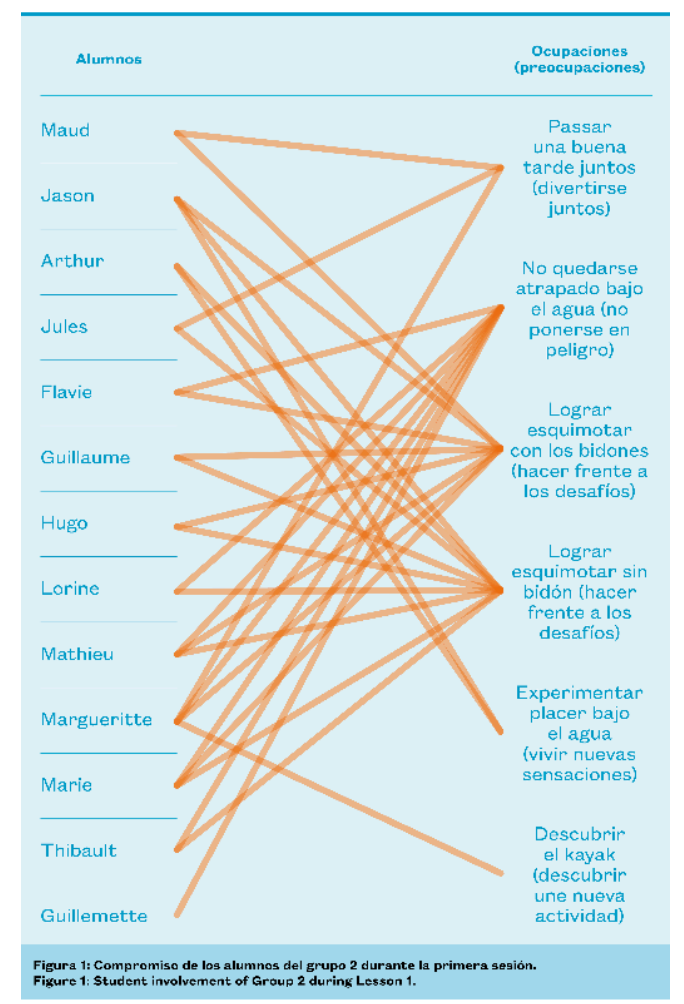

Figura 1 : Compromiso de los alumnos dei grupo 2 durante la primera sesión

Esta posibilidad de documentar, a partir de los relatos, el compromiso en términos de historias y de modos de involucramiento puede presentar intereses prácticos y epistémicos que no esbozamos aquí más que de manera especulativa. Para algunos profesores, los relatos dan un panorama rápido de todo aquello que inspira a los alumnos desde las primeras clases para alentarlos a continuar o transformar su compromiso. Proporcionan asimismo los materiales para investigar acontecimientos que generan, obstaculizan o aseguran la continuidad del compromiso.

\subsection{Relatos heurísticos para diferenciar los representamens de los alumnos}

Los relatos de experiencia resultaron también fructíferos para documentar representamens (o juicios perceptivos). De esta manera, pudimos identificar cómo los alumnos captaban lo que el entorno les ofrecía (ej., un kayak, un profesor, una compañera de equipo, un bidón, un faldón, el agua, etc.) Estos elementos constituían, desde el punto de vista del actor, oportunidades de acciones y tenían como particularidad, en los relatos, estar unidos a juicios perceptivos. Por ejemplo, en el relato de Anna durante la primera clase, el kayak era preponderante cuando ella lo golpeaba para alertar; el profesor, cuando ella observaba la demostración; sus compañeras de equipo, cuando la ayudaban; el bidón, cuando trataba de volver a la superficie; el faldón, cuando estaba bloqueada bajo el agua; el agua, cuando trataba de escapar de ella, etc.. Dicho de otra manera, los relatos de experiencia dan la posibilidad de completar los representamens sin reducirlos a su anclaje en un elemento del entorno. Sin embargo, aunque los relatos de experiencia permitían documentar de 
manera relativamente intensiva percepciones vinculadas a elementos externos al cuerpo (elementos naturales, materiales y humanos), lo permitían en menor medida para informar acerca de percepciones vinculadas al cuerpo (sensaciones musculares, de equilibrio, de esfuerzo, etc.).

Teniendo en cuenta la orientación de nuestra investigación que consistía en abordar los procesos de aprendizaje a través de la transformación del acoplamiento del actor y de su entorno, nos abocamos a dar cuenta de la evolución de los representamens sin separar los elementos captados en el entorno de los juicios perceptivos a los que dichos elementos estaban asociados.

\section{Los espacios de acción : la propuesta de un nuevo objeto teórico para explicar la transformación del acoplamiento actor-situación}

Junto con un perfeccionamiento del modo de procesar los relatos, hemos intentado definir un objeto teórico capaz de explicar la transformación del acoplamiento actorsituación.

\subsection{La definición de un objeto teórico para realizar un seguimiento de la evolución de los representamens}

En este momento, nos faltaba un objeto teórico para pasar en un momento dado de la constatación de los representamens (es decir, los juicios perceptivos) al análisis de su evolución y de su transformación a lo largo de las clases.

Para responder a estas necesidades redujimos el objeto teórico "curso de experiencia" al "espacio de acción". Este corresponde, para un actor, y por una unidad de tiempo determinada, al conjunto de los elementos del entorno a los que él atribuye cualidades particulares. Esta reducción presenta tres intereses mayores. El primero es tener una visión sintética del mundo propio de un alumno, sin distancia focal a priori sobre ciertos componentes del entorno (ej., los objetos, las relaciones con los otros alumnos, las relaciones con el profesor, los elementos naturales, etc.). El segundo interés es poder explicar la evolución de los juicios perceptivos de un alumno en un período largo de tiempo, distinguiendo diferentes unidades temporales durante un período de enseñanza-aprendizaje. En nuestro estudio, la comparación de los espacios de acción por sesión resultó pertinente en el sentido en que la sesión constituye, en EPS, una unidad de análisis suficiente como para comprender más globalmente el trabajo escolar en los cursos (Gal-Petitfaux, 2011). Es también un compromiso entre una unidad lo suficientemente estrecha como para captar la experiencia de los alumnos en kayak de mar sin discontinuidad (sin alternancia con otras prácticas), y lo suficientemente amplia como para seguir la pista de las evoluciones en un lapso largo de tiempo. El tercer interés es, cuando la documentación del espacio de acción se amplía a la escala del curso, poder captar lo que comparten o lo que potencialmente comparten los actores, construyendo una continuidad entre el entorno que constituye un conjunto de elementos públicos que los actores pueden potencialmente captar, y las situaciones de cada uno de los alumnos que constituyen los elementos significativos del entorno para cada uno de ellos. 


\subsection{Una gramática mínima para documentar los espacios de acción a partir de los relatos}

Nuevamente, se planteaba un desafío metodológico para homogeneizar la manera de documentar los representamens que permiten describir y explicar la evolución de los espacios de acción. En un primer momento, identificamos lo que el entorno ofrecía y que los alumnos habían captado (Cuadro 2, columna 1). Se trataba de elementos del entorno sobre los que se anclaban juicios perceptivos. En un segundo momento, identificamos las cualidades que los alumnos atribuían a lo que el entorno ofrecía (Cuadro 2, Columna 2). Se trataba de juicios perceptivos asociados a las acciones en curso. Estos juicios perceptivos remiten a emociones (por ej., el remo como signo de placer), a sensaciones (por ej., las corrientes como signo de dificultades para avanzar), a usos prácticos (por ej., la ola como signo de posible surf), a interpretaciones (por ej., la presencia del profesor como signo de falta de autonomía), y hacen que, elementos del entorno constituyen señales para el actor. El cuadro 2 describe, a título de ejemplo, el espacio de acción de Anna a partir de su relato de la primera sesión (Cuadro 1)

Cuadro 2 : Documentación dei espacio de acción de Anna durante la primera sesión

\begin{tabular}{|c|c|}
\hline Lo que el entorno ofrece & Cualidades de lo que el entorno ofrece (juicios perceptivos) \\
\hline Compañeras del equipo & $\begin{array}{l}\text { Como signo de referencia de nivel (inferencia) } \\
\text { Como signo de manipulación } \\
\text { Como signo de incomodidad }\end{array}$ \\
\hline Profesores & Como signo de una demonstración \\
\hline Kayaks & Como signo de un objeto para alertar \\
\hline Bidones & Como signo de ayuda para esquimotar \\
\hline Faldón & Como signo de obstáculo para salir del kayak \\
\hline Agua & Como signo de estrés \\
\hline Cuadro 2: Documentació & $\begin{array}{l}\text { io de acción de Anna durante la primera sesión. } \\
\text { eld of actions during Lesson } 1 \text {. }\end{array}$ \\
\hline
\end{tabular}

Cuadro 2 : Documentación dei espacio de acción de Anna durante la primera sesión

\subsection{El análisis de la evolución de los espacios de acción}

Esta homogeneización en la manera de documentar los representamens nos permitió, para cada uno de los alumnos, documentar y representar la evolución de su espacio de acción clase tras clase (ver Cuadro 3) 


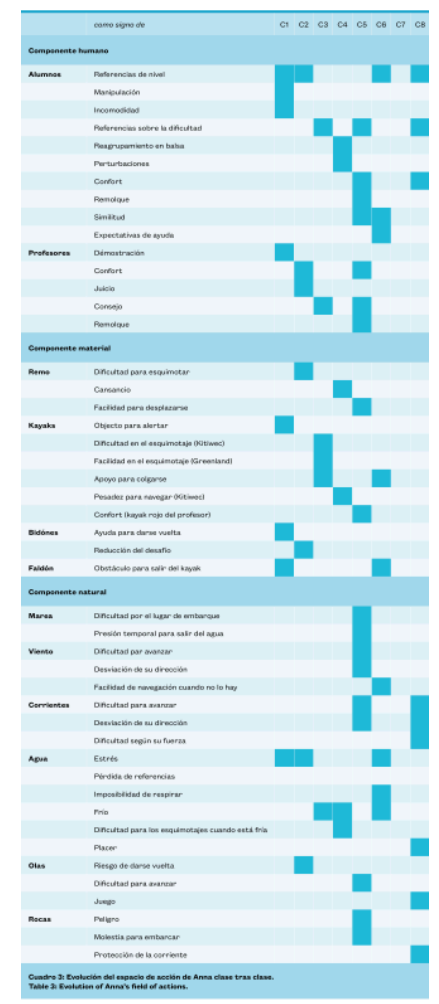

Cuadro 3 : Evolución dei espacio de acción de Anna clase tras clase cualidades molestas por cualidades colaboradoras. Esta transformación se pudo observar, por ejemplo, en las cualidades atribuidas al remo, inicialmente percibido como un objeto que estorbaba, que complicaba el esquimotaje y provocaba cansancio, 
luego, progresivamente percibido como una ayuda para desplazarse sin cansarse y sentirse "zen" en el agua. La segunda transformación típica es inversa : un elemento al que se le atribuían cualidades colaboradoras adquiría progresivamente cualidades más molestas. Esto se pudo observar en las cualidades atribuidas al profesor, cuyas intervenciones se percibían inicialmente como una ayuda para aprender los esquimotajes, luego, progresivamente como el signo de menor autonomía en el agua. Todas estas transformaciones son sostenidas por una transformación más global del mundo propio de los alumnos. Las cualidades atribuidas a un elemento del entorno no se transforman aisladamente, sino en coherencia con todo un conjunto de elementos significativos.

31 Por último, el análisis de los espacios de acción, enriquecido por una relectura de los relatos de experiencia, nos permitió distinguir dos categorías de cualidades compartidas por los alumnos. La primera se refiere a cualidades-prácticas. Permiten a los alumnos organizarse colectivamente. Esto fue observado por ejemplo en los niveles de soltura para manejarse atribuidos a ciertos alumnos. Al juzgar colectivamente que algunos alumnos se sentían más cómodos que otros en determinadas acciones, se distribuían los kayaks en función de su estabilidad, negociaban entre ellos un orden de paso en los talleres, se ponían de acuerdo sobre la mayor o menor dificultad que un paso presentaba, etc. La segunda categoría de cualidades compartidas se refiere a las cualidades-simbólicas. Estas daban a los alumnos la sensación de estar viviendo una aventura en común. Esto se observó por ejemplo en las cualidades atribuidas al remo eskimo. Al juzgar colectivamente que este remo correspondía específicamente al proyecto "Kayak ártico", se sentían parecidos y diferentes a los otros kayakistas con los que se cruzaban en el agua.

\section{La apropiación : un objeto de estudio pertinente para explicar la singularidad de los aprendizajes de los alumnos en kayak de mar, y más ampliamente la transformación del acoplamiento actor-situación durante los aprendizajes}

El Programa CdA propone explicar las transformaciones del acoplamiento acciónentorno en términos de apropiación. Se trata del "proceso de construcción de su propio cuerpo y de su propio mundo, insertando e incorporando principalmente elementos externos al acoplamiento actual, que luego se transforma" (Durand, 2013, p. 52, traducción libre). Durante los aprendizajes, el actor no solo se adapta a un entorno, sino que lo modifica. Para explicarlo, Theureau formula la hipótesis de un entramado de tres procesos: in-situación, in-corporación, in-culturación (Donin \& Theureau, 2019; Theureau, 2011). Esta hipótesis nos pareció pertinente para elevar el nivel de genericidad de nuestros resultados y, al mismo tiempo, interesante para discutir y precisar la descripción de la evolución de los espacios de acción.

In-situación es un primer proceso de apropiación. Es el "paso de un elemento del mundo tout court al mundo propio de un actor" (Donin \& Theureau, 2019, p. 8, traducción libre). Durante este proceso, los elementos del entorno pasan de un estatus de elemento no distinguido y no pertinente para el actor a un estatus de elemento distinguido, significativo y que perturba la actividad del actor (Durand, Poizat, \& 
Goudeaux, 2015). Surge del análisis de los espacios de acción en que los elementos del entorno entran en la situación del alumno, no de manera neutra y objetiva, sino con cualidades para el actor. Esto invita a pensar la diversidad de los alumnos y sus posibles dificultades en términos de adopción del entorno y no sólo de falta de adaptación a un mundo exterior. Sucede asimismo que los alumnos hacen emerger islotes de coherencia en los cuales se encuentra un equilibrio entre las cualidades asociadas a los diferentes elementos de su entorno. Se atribuyen cualidades a elementos del entorno (ej., una contracorriente percibida como una ayuda para franquear un obstáculo rocoso) teniendo en cuenta cualidades atribuidas a otros elementos (ej., un alumno percibido como una referencia en dificultad frente a ese obstáculo rocoso). Estas coherencias globales son constitutivas del proceso de in-situación. Tienen como particularidad ser autónomas respecto de un recorte artificial y a priori del entorno en dimensiones humanas y no humanas, naturales y no naturales.

In-corporación es un segundo proceso de apropiación. Consiste en "la integración, parcial o total, del objeto o de la herramienta o del dispositivo con el propio cuerpo del actor, es decir, con el conjunto de las acciones posibles de este actor, de tal manera que dicho objeto o dicha herramienta se vuelva transparente y pierda su autonomía en relación al actor" (Donin \& Thereau, 2019, p. 8, traducción libre). Si bien los relatos de experiencia son bastante incompletos para explicar esta transparencia, nos permitieron, sin embargo, aclarar cómo cualidades atribuidas a elementos del entorno se transforman antes de que estos últimos se vuelvan transparentes. La evolución de los espacios de acción muestra una in-corporación gradual que va de la confrontación, la evitación, la desconfianza y la percepción de obstáculos hacia la camaradería, el acercamiento, la confianza y la percepción de oportunidades para actuar. Se trata de un proceso abierto que tiende no sólo a transformar cualidades, sino también a delimitar cada vez más detalladamente circunstancias en las que dichas cualidades se perciben. Esto invita a percibir el entorno del alumno que aprende un poco menos como un reservorio de problemas por resolver y un poco más como un compañero en el que se trata de identificar valores de utilidad (Durand, Hauw, \& Poizat, 2015).

In-culturación es un tercer proceso de apropiación. Consiste en la integración de elementos del entorno a su cultura de acción que comparten también entre sí los miembros de comunidades de prácticas (Durand, Poizat, \& Goudeaux, 2015). Por medio de este proceso, el actor llega a captar elementos que le dan la oportunidad de afirmar su identidad individual o colectiva, y hasta transformar su identidad. Al proporcionar una visión sintética sobre los espacios de acción clase tras clase, nuestros resultados muestran que los alumnos no sólo hacen enactuar mundos propios satisfaciendo sus propias normas. Transforman también sus propias normas gracias a las cualidades que ellos atribuyen a los elementos que constituyen su propio mundo. El espacio de acción de un alumno es a su imagen tanto como él mismo es la imagen de su espacio de acción. Algunos juicios perceptivos (ej., cualidades lúdicas atribuidas a las corrientes) despiertan nuevas sensibilidades (ej., las ganas de encontrar corrientes para divertirse). A nivel colectivo, los alumnos pueden captar elementos que simbolizan sus ganas de pertenecer a la comunidad de práctica del kayak ártico. A cambio, algunas cualidades compartidas entre los alumnos (ej., alumnas consideradas como referentes) hacen emerger nuevas normas (ej., órdenes de paso, distribución del material, etc.) constitutivas de su cultura común. Esto invita a pensar las sensibilidades de los alumnos 
menos como rasgos estables que como construcciones vinculadas a las cualidades que estos últimos atribuyen progresivamente a los elementos de su entorno.

Es por ello que nuestro observatorio (los relatos de experiencia) y nuestro objeto teórico (los espacios de acción) tienen un valor heurístico en el sentido que permiten precisar ciertas hipótesis del Programa CdA.

\section{Conclusión : una apertura de posibles para la intervención y la investigación}

Esta investigación, por su observatorio y las perspectivas que aporta a los procesos de aprendizaje, puede abrir nuevos posibles en materia de enseñanza y de formación (Terré, Sève, \& Huet, 2020) que investigaciones futuras podrían poner a prueba.

Hemos subrayado principalmente, el interés de pensar el aprendizaje en términos de apropiación y no sólo de adaptación, los progresos en términos de cooperación con el entorno y no sólo de resolución de problemas, y la identidad como transformada por el espacio de acción y no ya constituida (Simondon, 2005). Estos puntos de vista abogan en favor de currículos de enseñanza [3] y de formación que no estén exclusivamente pensados en términos de acciones que se deben dominar (ej., las diferentes etapas de un esquimotaje, las diferentes etapas del momento de volver a enfrentar una corriente, la manera de orientar su remo para girar acercando o alejando el agua), sino también en términos de niveles de apropiación del entorno (ej., la construcción de una relación de equipo con el agua, la percepción más pormenorizada de las diferentes corrientes, la sensación del agua deslizándose por el remo). Para que esta visión del aprendizaje sea fecunda en el plano práctico, los profesores y formadores deben ser sensibilizados al tema, los que, mediando algunas precauciones, pueden facilitar el encuentro entre un "adentro" (ej., la experiencia de sentir que se deslizan sobre el agua sin hacer esfuerzo) y un "afuera" (ej., las corrientes que pueden encontrar en el entorno). Por ejemplo, nuestros resultados invitan a destacar "ayuditas" que pueden captar del entorno, y no sólo obstáculos a superar. Esto puede traducirse en consignas particulares : pedir a los alumnos que remonten una corriente a lo largo de una costa imponiéndoles una parada, a fin de que investiguen sobre potenciales refugios. Puede consistir también en restringir o contrastar el entorno de práctica: pedir a los alumnos que realicen esquimotajes en kayaks más o menos voluminosos para captar mejor sus cualidades. Nuestros resultados subrayan asimismo la importancia de eliminar elementos del entorno cuya apropiación por parte de los alumnos podría tornar imposibles acciones que los profesores juzgan eficaces, o, al contrario, posibles, acciones que estos últimos juzgan ineficaces. La elección de los profesores de suprimir el remo al principio del aprendizaje de esquimotaje lo muestra claramente. Sin esta proscripción, los alumnos tienden a sentir el remo como un apoyo posible en el agua (por lo tanto en hacer fuerza sobre él y hundirlo) mientras que lo que se espera es que el remo sólo sea utilizado suspendido como la prolongación de un cuerpo que flota sobre el agua.

La construcción del observatorio a partir de relatos de experiencia - uno de cuyos objetivos era establecer relaciones entre la investigación y la enseñanza- nos permite también prever ayudas para los profesores. Pensamos que ellos mismos tienen que transformarse en investigadores en sus propios cursos, no sólo para identificar comportamientos motores, sino también para investigar acerca del espacio de acción actualizado por sus alumnos. El recurso puntual a relatos de experiencia por parte de 
los alumnos puede brindarles ayuda en esto. Planteamos la hipótesis que estos relatos pueden enriquecer el espacio de acción de los profesores, tornar sobresalientes acontecimientos que pasan desapercibidos en el flujo de la clase (in-situación), ofrecer nuevas oportunidades de intervención (in-corporación) y aguzar su sensibilidad en las relaciones que mantienen los alumnos con el entorno (in-culturación). Estas pistas permanecen siendo especulativas. Constituyen una invitación a llevar a cabo otras investigaciones sobre los eventuales efectos transformadores de una introducción de los relatos de experiencia en la enseñanza.

\section{BIBLIOGRAFÍA}

Bolstad, R. (2011). From "student voice" to "youth-adult partnerships" : lessons from working with young people as partners for educational change. Wellington, Nouvelle-Zélande : New Zealand Council for Educational Research.

Cahour, B. (2013). L'expérience utilisateur au cœur des nouveaux systèmes de mobilité. Revue Télécom, Les systèmes de transport intelligents : vers une nouvelle mobilité, 170.

Colin, D. (2014). Analyse des pratiques d'écriture dans le discours des enseignants : enjeux didactiques : analyse de déclarations d'enseignants de CM2 et de 6e. Thèse, Université d'Orléans.

Depraz, N., Varela, F. J., \& Vermersch, P. (2011). À l'épreuve de l'expérience. Pour une pratique phénoménologique. Bucarest : Zeta Books.

Donin, N., \& Theureau, J. (2019). Construire une interprétation, de l'appropriation de la partition à la répétition générale. La préparation d'un concert par le chef d'orchestre Pierre-André Valade à la lumière de l'analyse d'activité. Revue musicale OICRM, 6(1), 1-36.

Durand, M. (2013). Construction of dispositions and development of human activity : a theoretical framework illustrated by the case of a novice manager. International Journal of lifelong education, 32(1), 39-55.

Durand, M., Hauw, D., \& Poizat, G. (2015). Apprendre les techniques corporelles : une introduction. In M. Durand, D. Hauw, \& G. Poizat (Eds.), L'apprentissage des techniques corporelles (pp. 9-24). Paris : PUF.

Durand, M., Poizat, G., \& Goudeaux, A. (2015). Individuation, pensée de la formation et technologie éducative. In J. Baillé (Ed.), Du mot au concept : l'individu (pp. 117-144). Grenoble : Presses universitaires de Grenoble.

Gal-Petitfaux, N. (2011). La leçon d'Éducation physique et sportive : formes de travail scolaire, expérience et configurations d'activité collective dans la classe. Contribution à un programme de recherche en anthropologie cognitive. Note de synthèse pour l'Habilitation à Diriger des Recherches, Université Blaise Pascal de Clermont-Ferrand.

Joubert, C. (2018). (Ré)écrire à l'école, pour penser et apprendre. Dossier de veille de l'IFÉ, 123.

Lani-Bayle, M. (2006). Taire et transmettre. Les histoires de vie au risque de l'impensable. Lyon : Chronique sociale. 
Mitra, D. (2009). Collaborating with students : building youth-adult partnerships in schools. American journal of education, 115(3), 407-436.

Ministère de l'Éducation Nationale (2015). Le socle commun de connaissances, de compétences et de culture, Décret n²015-372, 31 mars 2015.

Norman D. (1988). The design of everyday things. New-York : Basic Books.

Simondon, G. (2005). L'individuation à la lumière des notions de forme et d'information. Grenoble : J. Million (Krisis).

Terré, N., \& Jan, P.-D (2019). Apprentissage du risque en kayak de mer. In O. Petiot (Ed.), Le risque, Pour l'Action. Paris : Editions EP\&S.

Terré, N., Saury, J., \& Sève, C. (2013). Émotions et transformation des connaissances en éducation physique : une étude de cas en kayak de mer. eJRIEPS, 29, 27-58.

Terré, N., Sève, C., \& Huet, B. (2020). L'évolution de l'espace d'actions des élèves : une aide à la compréhension des apprentissages en Education Physique et Sportive. Une étude de cas réalisée avec des élèves de troisième en kayak de mer. eJRIEPS, 47.

Terré, N., Sève, C., \& Saury, J. (2016). La construction et le devenir des connaissances chez les élèves en éducation physique : une étude de cas réalisée au cours d'une séquence d'escalade. Revue STAPS, 113, 89-105.

Theureau, J. (2006). Le cours d'action : Méthode développée. Toulouse : Octarès.

Theureau, J. (2010). Les entretiens d'autoconfrontation et de remise en situation par les traces matérielles et le programme de recherche " cours d'action ». Revue d'anthropologie des connaissances, 4(2), 287-322.

Theureau, J. (2011). Appropriations 1, 2, 3. Journée Ergo-Idf, 16 juin 2011, CNAM, Paris.

Theureau J., \& Jeffroy, F. (1994). Ergonomie des situations informatisées. Toulouse : Octarès.

Varela, F., Thompson, E., \& Rosch, E. (1993). L'inscription corporelle de l'esprit. Sciences cognitives et expérience humaine. Paris : Seuil.

\section{NOTAS}

1. Golfo de la región de Bretaña en Francia.

2. Los seis componentes del signo hexádico se presentan más detalladamente en el capítulo 5 de este documento. [nota del traductor: las siglas se han dejado en Francès].

3. Un conjunto formalizado y ordenado de objetivos, de contenidos y de puestas en situación que estructuran la enseñanza.

\section{RESÚMENES}

El desafío de este artículo basado en un estudio llevado a cabo con un curso de 26 alumnos que formaron parte de un proyecto anual llamado "Kayak ártico", es doble. El primer desafío consiste 
en presentar la dinámica de construcción conjunta, a través de idas y vueltas, entre un observatorio y un objeto teórico. El segundo desafío es presentar nuevas nociones susceptibles de enriquecer el programa de investigación del "curso de acción". La primera de ellas es la noción de "relato de experiencia" que permite concebir un observatorio que apunte a abordar la experiencia de un colectivo importante de actores en un periodo de tiempo relativamente largo. Estos relatos ofrecen una descripción condensada de la experiencia del conjunto de los alumnos durante las clases de kayak que conducen a diferentes ejes de análisis. La segunda noción es la de "espacio de acción", que es una reducción del objeto teórico "curso de la experiencia". Corresponde, para un actor, y para una unidad de tiempo determinada, al conjunto de los elementos del entorno a los que ese actor atribuye cualidades particulares. El análisis de los espacios de acción de los alumnos permitió identificar evoluciones y transformaciones típicas en sus juicios perceptivos durante las clases. Estos resultados especificaron la hipótesis de los tres procesos (in-situación, in-corporación, in-culturación) de la apropiación propuesta por Theureau.

Este artigo, baseado num estudo realizado com uma turma de 26 alunos que faziam parte de um projeto anual denominado "Arctic Kayak", tem um desafio duplo. O primeiro desafio é apresentar a dinâmica da construção conjunta, através de voltas e reviravoltas, entre um observatório e um objeto teórico. $O$ segundo desafio é apresentar novas noções que possam enriquecer o programa de pesquisa do "curso de ação". A primeira delas é a noção de "relato de experiência" que permite conceber um observatório que visa abordar a experiência de um importante grupo de atores num período de tempo relativamente longo. Essas histórias oferecem uma descrição condensada da experiência de todos os alunos durante as aulas de caiaque que levam a diferentes eixos de análise. A segunda noção é a de "espaço de ação", que é uma redução do objeto teórico "curso de experiência". Corresponde, para um ator e para uma determinada unidade de tempo, ao conjunto de elementos do ambiente aos quais esse ator atribui qualidades particulares. A análise dos espaços de ação dos alunos permitiu identificar evoluções e transformações típicas nos seus julgamentos percetivos durante as aulas. Esses resultados especificaram a hipótese dos três processos (em situação, em corporação, em cultura) da apropriação proposta por Theureau.

L'enjeu de cet article, qui s'appuie sur une étude conduite avec une classe de 26 élèves engagés dans un projet annuel "Kayak arctique ", est double. Le premier est de présenter la dynamique de construction conjointe, par des allers et retours, entre un observatoire et un objet théorique. Le deuxième enjeu est de présenter des nouvelles notions susceptibles d'enrichir le programme de recherche du cours d'action. La première est la notion de “ récit d'expérience » qui permet de concevoir un observatoire visant à appréhender l'expérience d'un collectif d'acteurs important sur une temporalité relativement longue. Ces récits offrent une description condensée de l'expérience de l'ensemble des élèves au cours des leçons de kayak, ouvrant vers différents axes d'analyse. La deuxième notion est celle d'" espace d'actions ", qui est une réduction de l'objet théorique " cours d'expérience ». Il correspond, pour un acteur, et pour une unité de temps donnée, à l'ensemble des éléments de l'environnement auxquels il attribue des qualités particulières. L'analyse des espaces d'actions des élèves a permis de repérer des évolutions et des transformations typiques dans les jugements perceptifs des élèves au cours des leçons. Ces résultats ont précisé l'hypothèse des trois processus (in-situation, in-corporation, in-culturation) de l'appropriation proposée par Theureau.

The aim of this paper, based on a study conducted with a 26-student class engaged in an annual "Arctic Kayak" project, is twofold. The first challenge is to present the dynamics of joint construction, going back and forth between observation analysis and theoretical object. The second is to introduce two new concepts that might benefit the course-of-action research program. The first, "story of experience", helps designing and conducting observation research to collect and analyze the experience of large groups of actors over a relatively long period of 
time. Such stories offer a condensed description of students' individual experiences during the kayak classes, providing multiple angles of analysis. The second concept is "action field", which is a subset of the "course-of-experience" theoretical object. For an actor and a given unit of time, this corresponds to all elements of its environment to which it attributes characteristics. Analysis of the students' action fields allowed us to identify evolutions and transformations which are typical of the students' perceptual judgments during classes. These results clarify the hypothesis of the three processes (in-situation, in-corporation, in-culturation) of appropriation proposed by Theureau.

\section{ÍNDICE}

Mots-clés: cours d'action, kayak, récits d'expérience, espace d'actions, appropriation Keywords: course of action, kayak, story of experience, field of actions, appropriation Palabras claves: curso de acción, kayak, relatos de experiencia, espacio de acción, apropiación Palavras-chave: curso de ação, caiaque, histórias de experiência, espaço de ação, apropriação

\section{AUTORES}

\section{NICOLAS TERRÉ}

IFEPSA (UCO, Angers), APCoSS , 49 rue des Perrins, 49130, Les Ponts-de-Cé

nterre@uco.fr

\section{CAROLE SÈVE}

Université de Nantes, Laboratoire “ Motricité, Interactions, Performance » (EA 4334), 25 boulevard Guy Mollet,BP 72206, 44322, NANTES Cedex 03 carole.seve@igesr.gouv.fr

\section{BENOÎT HUET}

Université de Nantes, Laboratoire “ Motricité, Interactions, Performance » (EA 4334), 25

boulevard Guy Mollet, BP 72206, 44322 NANTES Cedex 03

Benoit.Huet@univ-nantes.fr 